\title{
An Energy Efficient Clustering-based Load Adaptive MAC (CLA- MAC) Protocol for Wireless Sensor Networks in IoT
}

\author{
Atul Kumar Pandey ${ }^{\mathrm{a}}$, Nisha Gupta ${ }^{\mathrm{b}}$ \\ ${ }^{a}$ Department of Electronics and Communication Engineering, Birla Institute of Technology, Extension Center \\ Patna, Patna-800014, India \\ ${ }^{b}$ Department of Electronics and Communication Engineering, Birla Institute of Technology, Mesra, Ranchi- \\ 835215 , India
}

Received: 08 June 2019; Accepted: 09 August 2019; Published: 08 September 2019

\begin{abstract}
Recent advancement in microelectronics and sensor devices have led to the development of a new family of networks called Internet of things (IoT). One of the challenges of IoT is the integration of autonomous and smart devices with multiple constraints specific to the wireless sensor network (WSN) environment. Among these constraints, the most important constraint is the energy efficiency of medium access control (MAC) protocol. To solve this problem, we propose an improved protocol called clustering based load adaptive MAC (CLA-MAC) for WSNs in IoT scenario, which involves mainly traffic load balancing within each cluster to provide a tuneable trade-off between energy consumption, delay, and throughput. The proposed solution makes the MAC protocol more energy efficient by reducing competition between sensor nodes with the adoption of clustering scheme and traffic distribution, which can significantly reduce the probability of collision and idle listening. Simulation results indicate that the proposed approach performs better compared with load adaptive MAC (LA-MAC) protocol in typical IoT scenario.
\end{abstract}

Index Terms: Internet of Things, Wireless sensor networks, Medium access control, Energy efficiency and clustering.

(C) 2019 Published b y MECS Publisher. Selection and/or peer review under responsibility of the Research Association of Modern Education and Computer Science

\footnotetext{
* Corresponding author. Tel.:

E-mail address: atulkrpandey@gmail.com
} 


\section{Introduction}

A recent development in processing, communication, and micro-electromechanical technology have spawned a considerable research interest in WSN designs for various applications. The new trend in the research field of sensor networks sets its emphasis on the network technology and information processing to make it appropriate for extremely high dynamic and resource-constrained networks. The integration of low cost, low power miniature components such as radios, sensors, and processors on a single chip, spawned a considerable interest in the field of WSNs from industrial and research viewpoints and thus various novel civilian applications such as environment monitoring, vehicular sensor networks, and body sensor networks have appeared [1].

The idea of the evolution of IoT came into existence parallelly with WSNs [1,2]. Unlike the IoT, the WSNs assume a specific technology and proliferate many applications in industries, medicals, and businesses, etc. The small, rugged, low-priced and low powered smart objects such as sensors, actuators, processors, etc. with multiple constraints specific to the wireless scenario enable the IoT to install the tiniest objects in any environment at the lowest price. Integrating such types of objects into IoT makes WSN more advance. Based on the infrastructure system, existing WSN applications are synthesized and potential new applications $[1,2]$ are identified and developed to fulfill the demands for future technology and market trends, such as for business, industry, medical, smart home, smart grid, smart water, and intelligent transportation systems generating a large amount of data that can be served to accomplish several purposes. Furthermore, as the modern world swings towards this innovative era of WSNs in the IoT scenario, several essential performance requirements like energy efficiency, scalability, small latency, and high concurrency are expected simultaneously. All these design constraints depend on several OSI layers but mainly focused on MAC layer [1-3].

The MAC layer performs channel accesses coordination, collision avoidance, scheduling of data packet transmission, and throughput maximization with an acceptable packet latency and energy consumption. The WSN-MAC protocols are classified broadly into two categories depending on the manner the access is being controlled [3]: reservation-based (i.e., TDMA based) and contention-based (i.e., CSMA based). In contentionbased access mechanism sensor devices are contending for a shared medium while in reservation-based access mechanism sensor devices get unique time slots, frequency channels or codes for data transmission. Contention-based protocols are not energy-efficient enough for real-time monitoring applications where a higher level of concurrency is required because as the data traffic increases the overall energy consumption will increase. On the other hand, the problem with reservation-based MAC protocols lies with clock synchronization, lack of scalability, and bandwidth utilization.

Several WSN applications in IoT scenario require higher concurrency with small payload length, limited energy, and limited capacity, will suffer from substantial control packet overheads and inefficient spectrum utilization. Therefore, the standalone application of existing WSN-MAC protocols would not be a suitable solution for real-time applications in IoT scenario. It shows the requirement to explore other MAC solutions for future WSNs in IoT scenario. Among all the design constraints as mentioned earlier the most important design constraint is energy efficiency because all the smart devices in the network are usually battery-powered and long battery life is critical for them especially in remote areas. Therefore, an energy efficient CLA-MAC protocol has been proposed in this paper, especially for monitoring applications in IoT networks to improve energy efficiency while maximizing the throughput and minimizing the delay. It performs the communication within the cluster by combining the benefits of existing MAC protocol with a clustering mechanism.

The remainder of this article is organized as follows: In the next section, related works are outlined. The design of the novel proposed CLA-MAC protocol is discussed in section III. Simulation results are presented in section IV and concluding remarks are presented in section $\mathrm{V}$ 


\section{Related Works}

Energy efficiency is the essential design constraint when dealing with the application, which requires higher concurrency. Idle listening, collisions, overhearing, and control packet overhead are the primary sources of energy consumption in the operation of MAC layer protocols. The MAC layer controls the energy consumption in sensor devices by adopting an appropriate channel access mechanism. A brief overview with regards to MAC protocols and clustering schemes for WSNs in IoT scenario is presented next.

The development of WSN based MAC protocols are studied in [3-9]. In this framework, IEEE 802.15.4 [4] is observed as one of the most appropriate technologies for WSNs research studies. In beacon-enabled mode, IEEE 802.15.4 introduces a super-frame structure, composed of Contention Access Period (CAP) and Guaranteed Time Slots (GTS). Operation in GTS is suitable for very low latency applications while CAP is preferred where higher scalability is required. In highly concurrent applications, operation in GTS is not suitable while CAP is found suitable to perform communication with the base station (BS). The multi-layer MAC (ML-MAC) [5] is a self-organized distributed contention-based protocol. It introduces a frame structure with active and sleep duration, where the active duration of each frame is divided into multiple nonoverlapping layers. It consumes a lesser amount of energy than Sensor-MAC (S-MAC) and (Timeout-MAC) TMAC protocol [3]. The asynchronous scheduled MAC (AS-MAC) [6] is an energy efficient, asynchronous, low power listening, and duty cycle MAC protocol in which wakeup time of neighbor sensor nodes are stored in an uncoordinated way and control packets are sent to each node during their wake-up times. To resolve sleep latency, packet delivery latency, synchronization overhead, and energy inefficiency; a receiver-initiated, wakeup time self-learning MAC (L-MAC) protocol was proposed in [7], where child nodes coordinate their wakeup time with their parent node. A schedule based multicast MAC protocol known as TRM-MAC was proposed in [8] to achieve low access delay and reliability, which utilizes the concept of Multicast Spanning Tree (MST). An energy efficient game theory based hybrid MAC (GH-MAC) protocol was proposed in [9], where game theory based TDMA (G-ETDMA) is used for outside-cluster communication and Game based nanoMAC (GnanoMAC) for inside-cluster communication to improve network lives. The development of WSN bases clustering algorithms was presented in [10], which categorizes the existing clustering techniques based on cluster design condition and cluster-head $(\mathrm{CH})$ selection parameters. Although the above discussed WSN-MAC protocols are providing higher energy efficiency, the standalone application of these protocols is not found suitable for the applications which demand high concurrent access, different quality of service (QoS) for sensor nodes, the existence of BSs to enable network assistance for improving device energy efficiency. This evokes the idea to explore other novel MAC solutions [11-21] which combines the benefits of MAC protocols with clustering schemes for future WSN applications in IoT scenario.

In this context, some standardization efforts have been made for diverse communication environments in recent years, similar to other standards such as Wireless-HART and IEEE 802.15.4. In [11], a network convergence model based on WSN and LTE-advanced network was developed where a simple energy saving model has been utilized for WSNs. Suitability of Random access channel (RACH) in LTE and LTE-Advanced for M2M communications is studied in [12]. In [13], a scalable hybrid MAC protocol has been proposed, where each communications frame is partitioned into contention and data transmission periods to reduce energy consumption by restricting the probability of collisions in data transmission period. The energy-efficient scheduling mechanism for machine nodes was investigated in [14] for cellular users together with LTE networks, and an energy-efficient MAC solution for direct communications between machine nodes and the BS to tackles massive concurrent access over a cellular network is also presented. In [15], the authors proposed a new MAC protocol for low power sensor nodes to achieve desired reliability and data rate requirements in a 5G wireless network for IoT systems. In [16], a routing technique was proposed to solve the energy-hole problem in IoT applications. MAC-DL [17] protocol was mainly designed to improve the quality of service and energy efficiency for typical IoT scenario significantly by considering the causes of frame losses for the proper 
functioning of the backoff algorithm. In [18], the performance of an IEEE 802.15.4 based multi-hop networks was evaluated in terms of channel congestion, reliability, and duty cycling. In [19], functionalities of MAC layer for low-power, low-rate, and, long-distance communication in three different technologies like LoRa, Sigfox, and NB-IoT were explored for smart cities applications. The Device-to-Device (D2D) communication is another emerging communication standard in the cellular network, which suggests an idea to collect and forward the traffic through D2D links [20]. In [21], authors provide an energy saving hybrid clustering protocol for Hy-IoT, green IoT and also for real-time IoT network architecture to improve nodes energy saving and simultaneously increasing the network living time as well as the data transmission rate.

As discussed earlier several promising MAC, and cluster formation algorithms are available in WSN. However, the direct applications of these protocols for WSNs in IoT scenario is not efficient due to the particular characteristics of WSN, which is assumed to be used in IoT. Therefore, achieving high concurrent access within the existing infrastructure of the IoT network in an energy-efficient manner is still an open problem and is the focus of this paper. The main contributions of this paper include, design of an energy efficient CLA-MAC protocol and impact of clustering on network design parameters such as energy, delay, throughput, and the probability of collision.

\section{Design of CLA-MAC Protocol}

Design of MAC protocol is influenced by many factors [1-3] as discussed earlier, but among all these design constraints scalability, energy efficiency, and high concurrent access require special consideration in IoT scenario. In WSNs, traffic congestion occurs due to the overload of traffic. Overcrowding traffic and congestion may cause packet loss, throughput degradation, and energy dissipation. Hence, a MAC protocol should also be designed with proper support for congestion control.

In the proposed CLA-MAC protocol, an effort is made to reduce the probability of collision, competition between sensor nodes and traffic load within the network to improve energy efficiency while maximizing the throughput and minimizing the delay. For this purpose, the concept of clustering is utilized in addition to the ML-MAC protocol [5]. Since the ML-MAC exhibits a load balancing characteristic; therefore, it can be defined as a load-adaptive MAC (LA-MAC) protocol for centralized access control, and hereafter, this ML-MAC is represented by LA-MAC. In the proposed CLA-MAC protocol, sensor nodes in the network are distributed into small separate regions to reduce the competition between adjacent sensor nodes and further each separate region is divided into several clusters to perform concurrent access using CSMA/CA mechanism.

The proposed algorithm operates in two modes: cluster formation and data transmission. Proposed CLAMAC protocol frame structure is portrayed in Fig.1, where time is divided into the setup phase (cluster formation period) and data transmission phase (data transmission period). Clusters are formed during the setup phase, and data transmission phase is used to perform data packet transmission which is further divided into several frames, and each frame is divided in to listen and sleep two periods as in the S-MAC [3] frame structure. The listen period is again partitioned into $\mathrm{M}$ non-overlapping access slots (ASs) where the time length of each access slot (AS) is equal. 


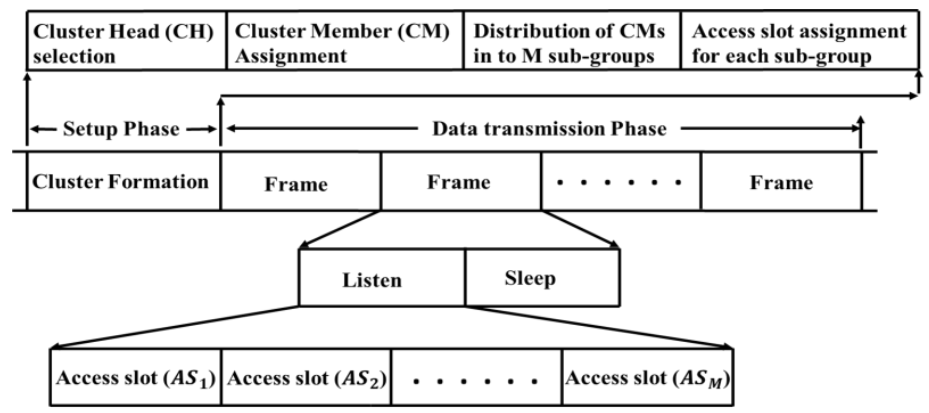

Fig.1. CLA-MAC frame Structure

\subsection{Cluster Formation}

The setup phase is used to form clusters in the network. Here, a simple static clustering algorithm is used. All sensor nodes within the network are uniformly randomly deployed in a square area. The complete network is divided into several small regions of equal area known as the grid. The BS is positioned at the center of the square region. Sensor nodes in each grid perform communication after forming clusters. The purpose of this physical partitioning is to form clusters as many as possible. This physical division is performed in such a manner so that the distance from sensor nodes to cluster heads $(\mathrm{CHs})$ and from $\mathrm{CHs}$ to $\mathrm{BS}$ can be reduced. The $\mathrm{CHs}$ status information can be transmitted directly to the BS or using any TDMA based mechanism. It is also considered that all the sensor nodes can perform communication within their own defined regions except $\mathrm{CHs}$. In each specific area, $\mathrm{CHs}$ are elected, and clusters are formed based on Low-Energy Adaptive Clustering Hierarchy (LEACH) protocol [22]. CHs are responsible for collecting the data in its specified region and sending it to the BS. The data transmission from $\mathrm{CH}$ to BS can be performed by utilizing a multi-hop routing mechanism. Such type of clustering provides full coverage and ensures proper utilization of energy. We have to try to form clusters as many as possible so that there are enough cluster heads to partake the traffic load of all the clustered nodes. However, it also increases access delay.

Initially, all the sensor nodes in the network are awake until cluster formation is done by selecting $\mathrm{CHs}$ in each grid and determining the members in each cluster. After cluster formation, all sensor nodes within each cluster is further divided into $M$ sub-groups. A random AS between 1 to $M$ is elected and assigned randomly for the sensor nodes in each sub-group. All cluster nodes within a sub-group are allowed to wake up and perform data transmission within their assigned AS only, using the CSMA/CA technique. Therefore, energy efficiency is improved by reducing the competition between sensor nodes within each cluster due to network partitioning and clustering.

\subsection{Data Transmission}

In CLA-MAC protocol, data transmission will start after completion of the cluster formation process. When a new sensor node connects with the network, after forming the cluster it selects an AS randomly within its cluster and broadcasts its schedule to the corresponding $\mathrm{CH}$. Each sensor node keeps the schedule of other sensor nodes in its schedule table to know the listening time of other sensor nodes that are working in different AS. To improve the network performance, high concurrent access is performed in each cluster independently with other clusters. That means a parallel access control is performed within each cluster locally. Therefore, cluster nodes only in the same AS will compete with each other during the data transmission stage. Thus, the number of sensor nodes in each AS decreases and competition between them is reduced and when the number 
of ASs becomes larger, single AS may be allocated for a single sensor node which results, a decrease in the probability of packet collision and some cases it approaches zero. To save the CHs energy consumption, member nodes within a sub-group of each cluster wishes to transmit a data packet will wake-up according to the schedule of their corresponding CHs. It transmits the data instead of changing the $\mathrm{CH}$ schedule by the schedule of cluster members in each sub-group, which makes $\mathrm{CH}$ stay awake while their members are transmitting and receiving their information. The $\mathrm{CH}$ node starts sleeping if no activity occurs in the AS. Then, $\mathrm{CH}$ sends their received data to the BS in a scheduled and single hop manner while the member nodes are in the sleep mode. Therefore, the probability of collision is reduced due to clustering and traffic load distribution among M-ASs, which improves network performance in terms of throughput and energy efficiency.

\subsection{CLA-MAC protocol design analysis}

Based on the previous discussions, a cluster based load adaptive MAC solution (CLA-MAC) has been proposed with an emphasis on energy savings and extension of battery lives. The concept of the proposed hybrid MAC protocol is originally inspired by IEEE 802.15.4 [4] and ML-MAC [5]. This section introduces how the proposed CLA-MAC protocol is designed by integrating the existing MAC and clustering protocols. Fig.1 shows the frame structure of the proposed CLA-MAC protocol. Since clustering is going to be implemented in the CLA-MAC protocol, frames in data transmission period should be divided into two parts: one for the intra-cluster communication within clusters, between cluster members and their corresponding $\mathrm{CHs}$ in listen period, and the other for the inter-cluster communication, between $\mathrm{CHs}$ and base station in the sleep period. The communication protocol required for intra-cluster communication needs to be scalable and adaptive to the changes in the network. Among all the protocols in literature, non-persistent version of CSMA/CA mechanism is found to be more suitable for inside-cluster transmission as it does not require extra control packet overhead and provides higher scalability. Energy consumption of non-persistent CSMA/CA grows as the sensor nodes become more, not only because the amount of data to send but also because denser traffic causes longer back-off time and more collisions. On the other side, TDMA based protocols have the best energy performance and don't lose in packet delay and throughput. However, it provides weak scalability. Therefore, only TDMA based protocols which consume the least energy is suitable for inter-cluster communications. An energy saving MAC mechanism to perform communication within every cluster is considered here in this paper. Next, the CLA-MAC protocol is introduced to overcome the drawbacks and further reduce the energy consumption of the inside-cluster network. In CLA-MAC idle listening is reduced by splitting listen period into M non-overlapping ASs. In each slot, only a small part of sensor nodes defined by each sub-group in every cluster is permitted to contend for the channel. Before each AS comes, the designated sensor nodes keep sleeping instead of listening. Since sleep power is minor to listen power, the scheme can decrease the total energy waste. However, as a consequence of trade-off, the delay of individual sensor nodes is increased. In the next subsection, energy consumption, delay and throughput analysis of CLA-MAC protocol are investigated.

\subsubsection{Energy Consumption}

Among all the versions of CSMA/CA techniques, non-persistent CSMA/CA technique is selected here for the analysis of CLA-MAC protocol due to its simplicity and low-cost operation. In nonpersistent CSMA/CA, a node wishes to transmit a packet first sense the channel, and if the channel is sensed to be busy, it waits for a random amount of time and repeats this process until the channel is 
found to be free for transmitting the data. In the following, we analyse the energy consumption in CLA MAC protocol based on non-persistent CSMA/CA technique [23].

Consider parameter $\lambda$ to define aggregate packet arrival rate of each node in the network, which includes both new arrivals and retransmitted ones. For the simplicity of the analysis, a separate channel is assumed for the transmission of acknowledgment packets. The channel is modeled as two-state Markov process with idle and busy states. Transmission can be either successful or failed in the busy state. Based on this model, both the transition probabilities between these two states are equal to 1 and probabilities that a channel is sensed idle or busy are equal to $P_{I}=P_{B}=0.5$. The average time spends in idle state is defined by the average time elapsed between two successive packets, i.e., $B_{I}=1 / \lambda$. Define $\tau_{p}$ as transmission delay and $\delta$ as propagation delay. The average duration of the busy period is $B_{B}=$ $\tau_{p}+\delta+\widehat{Y}$, where $\widehat{Y}$ represents the average time at which the last colliding packet is scheduled during a transmission period which begins at time $0 . \widehat{Y}^{\wedge}$ is calculated as follows [23]:

$$
\begin{aligned}
& F_{Y}(y)=\text { prob. }(\text { no arrival during } \delta-y)=e^{-\lambda(\delta-y)} \\
& \widehat{Y}=\delta-\left(1-e^{-\lambda \delta}\right) / \lambda
\end{aligned}
$$

A packet will be transmitted successfully if it starts after an idle period, and during that, other sensor nodes are not starting their transmission. Time-averaged idle channel probability is defined by the probability that the channel is sensed to be idle after the arrival of a new packet in the network and is calculated as follows:

$$
p_{i}=\frac{P_{I} B_{I}}{P_{B} B_{B}+P_{I} B_{I}}=\frac{1 / \lambda}{1 / \lambda+T-\frac{\left(1-e^{-\lambda \delta}\right)}{\lambda}}=\frac{1}{\left(\lambda T+e^{-\lambda \delta}\right)}
$$

Where $T=\tau_{p}+2 \delta$. Also, the probability of successful packet transmission if it starts after an idle period and not any other node schedules their packet transmission during it, i.e., no packet is scheduled during $\delta$ period is derived as $p_{t r}=e^{-\lambda \delta}$. Therefore, the probability of successful packet transmission is calculated by multiplying time-averaged idle channel probability $p_{i}$ with the probability of no transmission during $\delta$ period $p_{t r}$, as follows:

$$
p_{s}=p_{i} \times p_{t r}=\frac{e^{-\lambda \delta}}{\left(\lambda T+e^{-\lambda \delta}\right)}=\frac{1}{\left(\lambda T e^{\lambda \delta}+1\right)}
$$

Therefore, the average energy consumption in non-persistent CSMA/CA technique in the network is calculated as:

$$
\begin{aligned}
& E_{\text {Cons }}=\left(1-p_{i}\right) E_{\text {Idle }}+p_{i}\left(1-p_{s}\right) E_{\text {Coll }}+p_{s} E_{\text {Succ }} \\
& E_{\text {Succ }}=P_{t} \tau_{p}+P_{l} \tau_{r} \\
& E_{\text {Coll }}=E_{\text {Succ }}+P_{l} \sigma_{\text {Coll }}=P_{t} \tau_{p}+P_{l}\left(\tau_{r}+\sigma_{\text {Coll }}\right) \\
& E_{\text {Idle }}=P_{l} \sigma_{\text {Idle }}
\end{aligned}
$$

Where, $E_{\text {Idle }}, E_{\text {Coll }}$, and $E_{\text {Succ }}$ is defined as energy consumption during back-off, collision, and successful transmission, respectively. Also, $\sigma_{\text {Idle }}, \sigma_{\text {coll }}$, and $\tau_{r}$ are defined as average back-off after busy sensed channel, average back-off after the collision and, the round-trip-time delay from successful 
packet transmission to the acknowledgment packet arrival, respectively. Define, $P_{t}$, and $P_{l}$ as the power consumed in transmit and listen modes for the $i^{\text {th }}$ node, respectively.

In CLA-MAC protocol, the listen period is composed of $\mathrm{M}$ number of ASs, and in each AS nonpersistent CSMA/CA access technique is used to perform communication. Therefore, the traffic load in each AS will be reduced by a factor $\mathrm{M}$ and defined as $\lambda_{m}=\lambda / M$. Therefore, the energy consumption in the network using CLA-MAC protocol is derived here from (4)-(7) as:

$$
E_{\text {Cons }}=\frac{E_{\text {Succ }}}{1+\lambda_{m} T e^{\lambda_{m} \delta}}+\frac{\lambda_{m} T e^{2 \lambda m \delta} E_{C o l l}}{\left(1+\lambda_{m} T e^{\lambda_{m} \delta}\right)^{2}}+\left(1-\frac{e^{\lambda_{m} \delta}}{1+\lambda_{m} T e^{\lambda_{m} \delta}}\right) E_{\text {Idle }}
$$

\subsubsection{Delay}

Packet delay is defined as the time interval between the arrival instant of the packet and the time when it is successfully received by the receiver. Hence, before the successful transmission of a packet, the average time spent in contention, i.e., back-offs and retransmissions are considered to calculate the average delay in packet transmission. Therefore, the value of average total delay $\mathrm{E}\left[T_{D l y}\right]$ can be calculated as $\mathrm{E}\left[T_{D l y}\right]=T_{\text {Succ }}+\mathrm{E}\left[T_{\text {Cont }}\right]$. Where, $\mathrm{E}\left[T_{\text {Cont }}\right]$ is the average total contention time, and $T_{\text {Succ }}$ is the duration of a successful transmission, which has a constant value that depends on the access method used by the sensor nodes, and it can be calculated as $T_{\text {Succ }}=\tau_{p}+\tau_{r}$. Because the contention time of any packet is independent of the contention time of any other packet, the value of $\mathrm{E}\left[T_{\text {cont }}\right]$ can be calculated as $\mathrm{E}\left[T_{\text {cont }}\right]=k \mathrm{E}\left[T_{C}\right]$. Where $\mathrm{E}\left[T_{C}\right]$ is the average contention time required to transmit a packet and $k$ is the number of required retransmissions. The contention between successive successful transmissions is composed of several idle and collided slots of different durations. Therefore, the average number of slots required to achieve a successful transmission denoted by $\mathrm{E}[X]$ can be calculated as:

$$
\mathrm{E}[X]=\sum_{k=0}^{\infty}(k+1)\left(1-p_{s}\right)^{k} p_{s}=\frac{1}{p_{s}}
$$

Then, the total contention time is equal to $\mathrm{E}\left[T_{C}\right]=(\mathrm{E}[X]-1) . \mathrm{E}\left[T_{\text {Slot } \mid \text { un_Success }}\right]$, Where $(\mathrm{E}[X]-1)$ is the average number of unsuccessful slots before having a successful transmission and $\mathrm{E}\left[T_{\text {Slot } \mid \text { un_Success }}\right]$ is the average duration of a slot given that the slot is not successful. A slot is not successful if it either idle or collided. The average duration of any unsuccessful slot can be expressed as:

$$
\mathrm{E}\left[T_{\text {Slot } \mid \text { un_Success }}\right]=\left(\frac{p_{b}}{1-p_{s}}\right) \sigma_{\text {Idle }}+\left(\frac{p_{c}}{1-p_{s}}\right) T_{\text {Coll }}
$$

Where, $p_{b}$ and $p_{c}$ are the probabilities of back-off and collision, respectively, which can be calculated as $p_{b}=\left(1-p_{i}\right)$ and $p_{c}=p_{i}\left(1-p_{t r}\right)$. The probability of unsuccessful transmission due to a busy sensed channel and collision are $p_{b} /\left(1-p_{s}\right)$ and $p_{c} /\left(1-p_{s}\right)$ respectively. The duration of a collision $T_{\text {Coll }}$ depends on the access method, and it can be written as $T_{\text {Coll }}=\tau_{p}+\tau_{r}+\sigma_{\text {coll }}$. Therefore, the average total contention time can be rewritten as

$$
\mathrm{E}\left[T_{\text {Cont }}\right]=k\left(\frac{1}{p_{s}}-1\right)\left[\left(\frac{P_{b}}{1-p_{s}}\right) \sigma_{\text {Idle }}+\left(\frac{P_{c}}{1-p_{s}}\right) T_{\text {Coll }}\right]
$$

Hence, the average delay in packet transmission can be calculated as follows: 
$\mathrm{E}\left[T_{D l y}\right]=T_{\text {Succ }}+k\left(\frac{1}{p_{s}}-1\right)\left[\left(\frac{P_{b}}{1-p_{s}}\right) \sigma_{I d l e}+\left(\frac{P_{c}}{1-p_{s}}\right) T_{C 0 l l}\right]$

Also, the average packet delay for CLA-MAC is derived from (12) as follows:

$$
\mathrm{E}\left[T_{D l y}\right]=T_{\text {Succ }}+k\left(\frac{1}{\widehat{p_{s}}}-1\right)\left[\left(\frac{\widehat{p_{b}}}{1-\widehat{p_{s}}}\right) \sigma_{I d l e}+\left(\frac{\widehat{p_{c}}}{1-\widehat{p_{s}}}\right) T_{C 0 l l}\right]
$$

Where,

$$
\widehat{p_{l}}=\frac{1}{M} \times \frac{1}{\lambda_{m} T+e^{-\lambda} \delta}, \quad \widehat{p_{t r}}=e^{-\lambda_{m} \delta}, \widehat{p_{s}}=\widehat{p_{l}} \times \widehat{p_{t r}}, \widehat{p_{b}}=\left(1-\widehat{p_{l}}\right) \text { and } \widehat{p_{c}}=\widehat{p_{l}}\left(1-\widehat{p_{t r}}\right) .
$$

\subsubsection{Throughput}

The throughput of the network is defined as the fraction of time in which successful transmission happens. The throughput of the network for CLA-MAC is derived as follows:

$$
S_{T h p}=\frac{P_{B} \tau_{p} \hat{p}_{S}}{P_{B} B_{B}+P_{I} B_{I}}=\frac{\lambda_{m} e^{-\lambda m \delta} \tau_{p}}{\lambda_{m} T+e^{-\lambda} \delta}=\frac{\lambda_{m} T+e^{-\lambda m \delta}-1}{1+\lambda_{m} T e^{\lambda_{m} \delta}}
$$

\section{Simulation Results}

The performance of the proposed CLA-MAC protocol is evaluated through MATLAB computer simulation. The simulation results of the proposed CLA-MAC protocol were compared with simple LA-MAC, which is a non-clustered version of CLA-MAC protocol. To perform more perfect comparisons, all the assumptions are assumed the same as given in [5] for the simulation of CLA-MAC protocol. Table 1 illustrates the parameters and their corresponding elected values to perform the simulation.

Table 1. Simulation parameters and values

\begin{tabular}{llll}
\hline Parameter & Value (Unit) & Parameter & Value (Unit) \\
\hline Average inter-arrival time $T_{I A T}$ & $2-10$ second & Transmit Power $P_{T x}$ & $24.75 \mathrm{~mW}$ \\
Number of access slots M & $1-10$ & Listen Power $P_{L}$ & $13.5 \mathrm{~mW}$ \\
Number of sensor nodes N & 100 & Sleep power $P_{S}$ & $15 \mu \mathrm{W}$ \\
Frame duration $T_{F}$ & 1 second & Simulation time $T_{S}$ & 200 second \\
Access slot duration $T_{A S}$ & $0.3 / \mathrm{M}$ second & Sensor network Area A & $150 \times 150$ meter $^{2}$ \\
\hline
\end{tabular}

The simulation was performed for 200 seconds, and several sensor nodes were taken 100 for all the ten simulation runs. To perform the simulation, data packet length of each packet was considered 38 bytes and inter-arrival time $T_{I A T}$ between two consecutive packets ranges from 2 to 10 second. Simulation is performed ten times for different topologies, and the average of all the ten runs was taken to calculate the accurate results. 


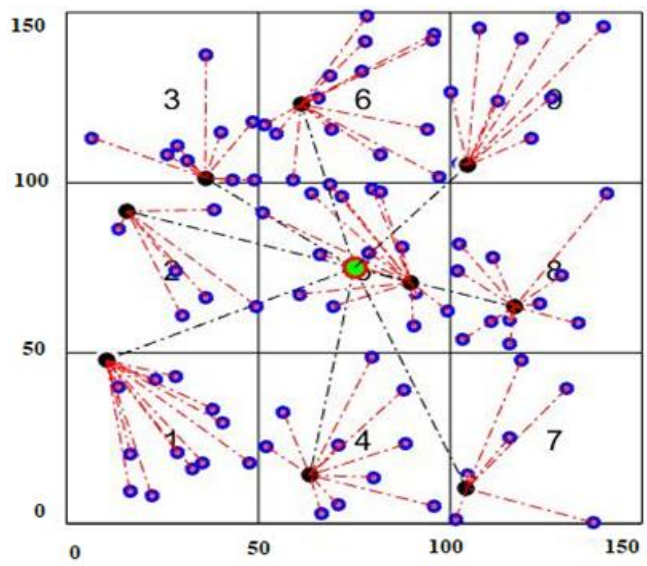

Fig.2. The simulated architecture of WSN in the IoT scenario

Fig. 2 shows the architecture of WSN in the IoT scenario with single $\mathrm{CH}$, which was simulated in MATLAB environment. All the sensor nodes were distributed randomly within a square region of 150 $\mathrm{x} 150$ meter $^{2}$ dimensions, and BS was placed at the center of this square region. The square region is further divided into nine small equal size regions known as a grid to facilitate clustering. Clustering is used to form many clusters as possible in each small size grid. To form the static clusters in each grid original LEACH algorithm was slightly modified and the number of $\mathrm{CHs}$ are selected based on the probability of $\mathrm{CH}$ selection $p_{C H}$ similar to original LEACH algorithm. Fig.2 shows the network architecture with single $\mathrm{CHs}$ in each grid that was simulated for the performance analysis of the proposed CLA-MAC protocol. As indicated in Fig.2, CHs were marked with a black color, cluster members (CMs) were marked with a blue color, and BS is marked with green color.

The main advantage of the proposed hierarchical CLA-MAC protocol is that it reduces the overall competition between all sensor nodes within each cluster in the network, which reduces the probability of collision and improves the network performance. Therefore, the probability of successfully transmitted packets in the proposed CLA-MAC protocol is significantly improved, which will be discussed later in the next subsection. The performance efficiency of CLA-MAC protocol is validated by comparing it with LA-MAC (CLA-MAC without clustering) protocol concerning several design parameters like throughput, delay, energy consumption, and the probability of collision.

\subsection{Throughput analysis}

Throughput of the network is defined by finding the number of successfully transmitted data packets per unit time. In CLA-MAC protocol, all the sensor nodes in each cluster are randomly distributed into $M$ sub-groups, and these sub-groups are mapped with $M$ number of AS through one-to-one mapping. The communication between sensor nodes in each sub-group of a cluster (CMs) to the $\mathrm{CH}$ is performed by using the CSMA/CA mechanism. Since in clustering based proposed CLA-MAC protocol, a single separate ASs are assigned to each sub-group of sensor nodes among all the M-ASs in each cluster of a grid. Hence, it is possible to assign a single AS to each node as the number of ASs becomes higher similar to the TDMA based MAC protocol. In this way, the competition between sensor nodes in a cluster is reduced, and the number of generated packets will be transmitted successfully, which improves the network throughput. In comparison to CLA-MAC protocol, the LA-MAC protocol requires less number of ASs and allows more sensor nodes to access an AS. This results in high 
competition between sensor nodes in each AS in the network, and fewer sensor nodes will get the chance to transmit their data packets, which reduces the throughput performance of LA-MAC concerning CLA-MAC.

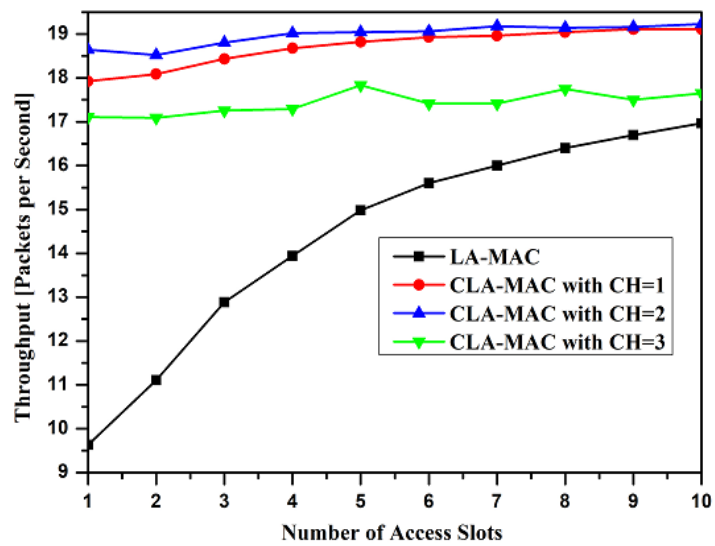

Fig.3. Average throughput with $T_{I A T}=5$ second

Fig. 3 shows the throughput performance of CLA-MAC concerning a variable number of ASs at a fixed message inter-arrival rate $\lambda$ of 0.2 corresponding to a fixed value of message inter-arrival time $T_{I A T}$ of 5 seconds. Hence, a total of 40 packets will be generated during the simulation time of 200 seconds. Fig. 3 also illustrates the significant difference in throughput performance with and without clustering.

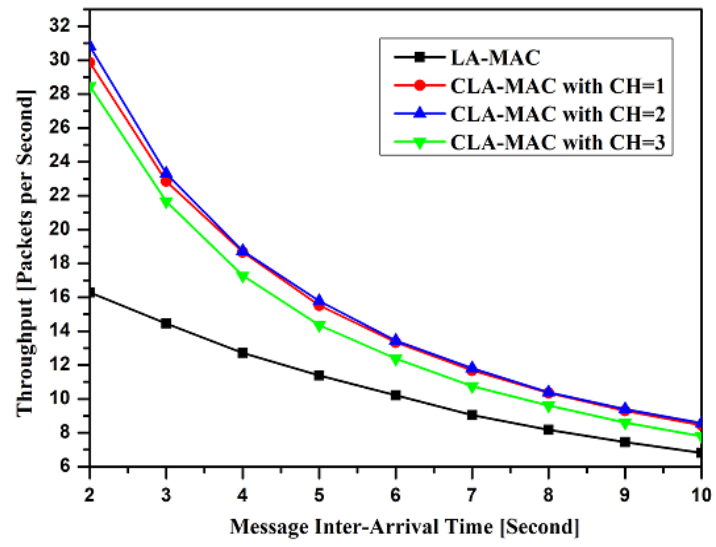

Fig.4. Average throughput with $\mathrm{AS}=3$

The effect of message inter-arrival time $T_{I A T}$ at a fixed value of AS on throughput performance of LA-MAC and CLA-MAC with different number of $\mathrm{CHs}$ is also represented in Fig.4. The number of ASs is assumed to be fixed with a value equal to three. Traffic load $\lambda$ is inversely proportional to message inter-arrival time $T_{I A T}$. Hence, an increase in message inter-arrival time $T_{I A T}$ indicates a corresponding decrease in traffic load and vice-versa. The probability of collision increases as the traffic load becomes high in both LA-MAC and CLA-MAC protocols. Therefore, less number of sensor 
nodes will be allowed to transmit the data packets. Also, as the traffic load increases, the probability of collision in CLA-MAC protocol decreases in comparison to LA-MAC due to clustering. The probability of successfully transmitted packet in CLA-MAC protocol increases as well, which results in an increase in throughput in comparison to LA-MAC. Fig.3 and Fig.4 also show that as the number of CHs are increased up to two clusters in each grid, the throughput performance of CLA-MAC is further improved but as the number of the cluster becomes three, throughput is reduced because fewer number of sensor nodes are available in each cluster to send the data packet. Nevertheless, we observed that the proposed CLA-MAC has better performance in contrast to the LA-MAC protocol due to the utilization of load partitioning and hierarchical clustering in CLA-MAC.

\subsection{Energy consumption analysis}

As portrayed in Fig.3 and Fig.4, the number of successfully transmitted packets in the CLA-MAC protocol was more than LA-MAC protocol. Thus, it can be said that the energy consumption of the proposed CLA-MAC protocol is higher. The total energy consumption of each node is obtained by the sum of energy spent to listen, transmit, and sleep mode. Fig.5 compares the average energy consumed by a node in LA-MAC and CLA-MAC protocols.

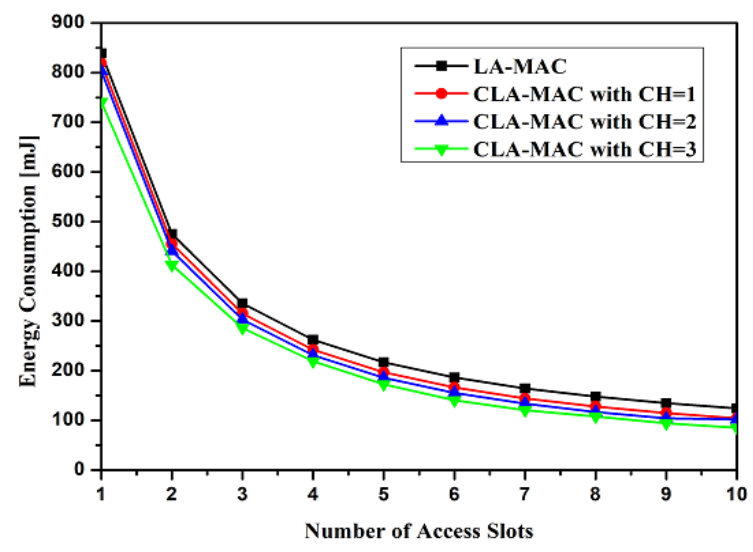

Fig.5. Average energy consumption with $T_{I A T}=5$ second

It is clear from the graph as given in Fig. 5 that for the small number of clusters in each grid such as one or two the energy consumption in both protocols are similar because energy consumption in transmission mode is higher in CLA-MAC than LA-MAC due to the transmission of more number of successful packets than LA-MAC. However, in LA-MAC protocol number of unsuccessful transmissions are larger than CLA-MAC, which results in more energy consumption in idle listening and retransmission of packets due to collisions. Therefore, in the proposed CLA-MAC protocol, more packets are transmitted in comparison to LA-MAC with approximately equal energy consumption. Also, as the number of clusters is increased (for $\mathrm{CH}=3$ ) in each grid, energy consumption in CLA-MAC will be reduced because it works as a TDMA based protocol. Lesser number of sensor nodes in each AS reduces the competition between sensor nodes and improves the delay performance of the network in CLA-MAC protocol. Energy consumption in both protocols is decreased with an increase in the number of ASs. 


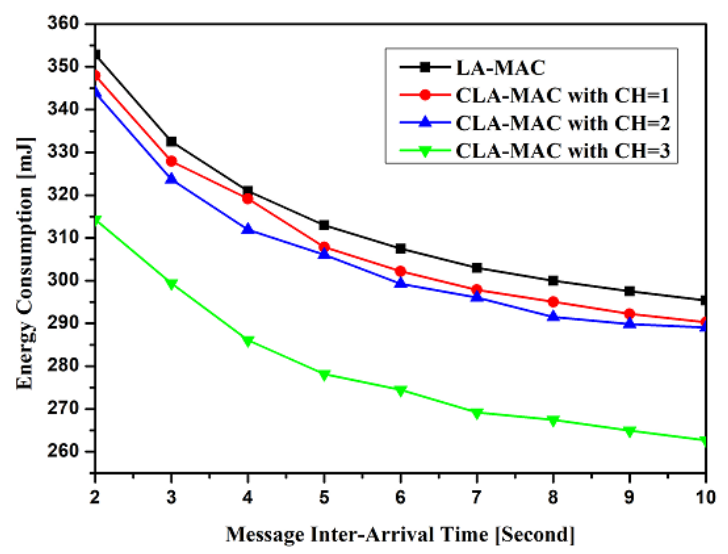

Fig.6. Average energy consumption with $\mathrm{AS}=3$

Fig.6 illustrates the average energy consumption for each node with different message inter-arrival times. The number of ASs, in this case, was taken to be three. Since it sent more packets at high traffic, the energy consumption of the proposed CLA-MAC algorithm was higher than the LA-MAC protocol in transmitting state. But the energy consumption due to idle listening and retransmission is less in CLA MAC in contrast to LA-MAC. Therefore, the overall average energy consumption in CLA-MAC is less than LA-MAC. Also, the energy consumption in both protocols decreases by reducing the traffic.

\subsection{Delay analysis}

Delay in a packet transmission is referred to as total time elapsed since it is stored in the transmission buffer until it is successfully received at the receiver. It includes queuing delay that a packet may encounter in the buffer during a sleep mode of a node and access delay that is the time a node waits until it's AS enables. It is also clear that the access delay is negligible compared to queuing delay.

Packets encounter less delay during the transmission with less number of ASs and in contrast, as the number of ASs increases packet delay increases. Fig.7 shows the average delay performance of both CLA-MAC and LA-MAC protocols under a constant traffic load of 0.2 with variable ASs and Fig. 8 also shows the delay performance of both protocols at a constant ASs equal to 3 with variable traffic load. As the probability of packet transmission increases packet delay increases. Thus, the delay in the proposed CLA-MAC protocol is higher than the LA-MAC protocol. Further delay in CLA-MAC can be reduced by forming more number of clusters in each grid. 


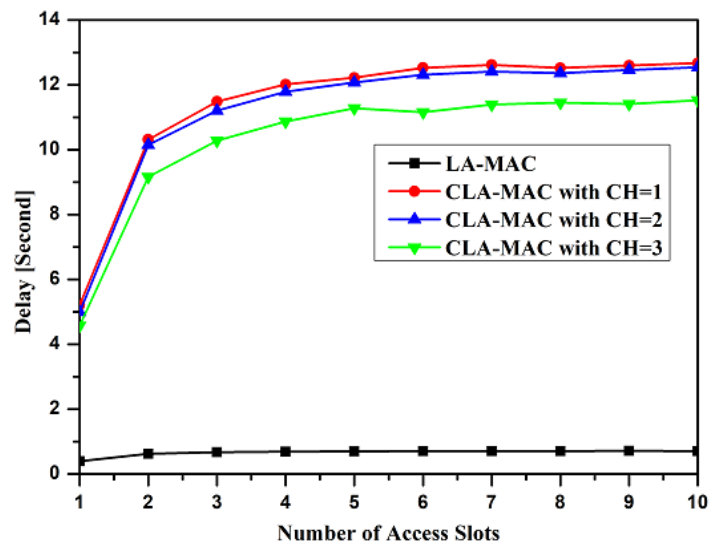

Fig.7. Average delay with $T_{I A T}=5$ second

Proposed load-adaptive CLA-MAC protocol divides listen interval into M-ASs and allows only a portion of CMs to compete for channel access in each AS. Before the start of allocated AS each node in a cluster are in sleep state instead of listening, and newly arrived packets are buffered. It is clear that for the value of $\mathrm{M}$ equals to one CLA-MAC behaves similar to conventional CSMA/CA and when the value of $M$ is sufficiently large, at most one node will be assigned to each AS and it behaves like a scheduling-based MAC.

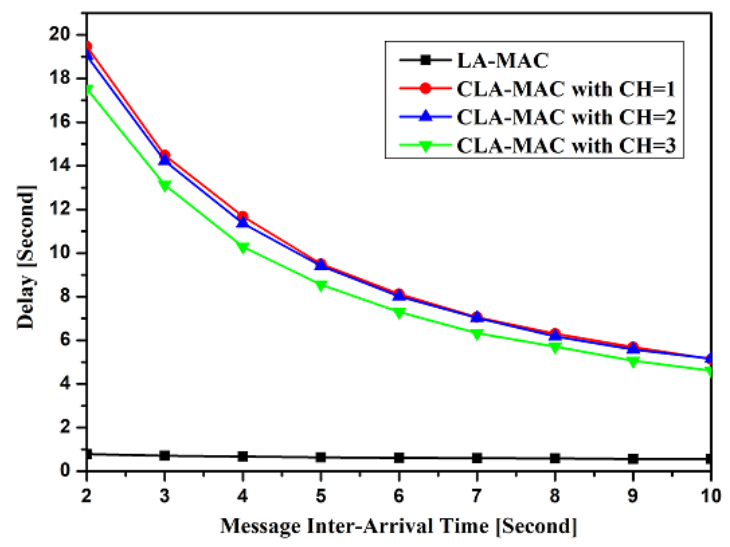

Fig.8. Average delay with AS $=3$

Therefore, CLA-MAC with M-ASs using CSMA/CA in each AS provides a trade-off between CSMA-based and TDMA-based MAC protocols. The desired level of energy efficiency can be achieved by reducing idle listening time and the probability of collisions both while the increasing probability of successful packet transmission simultaneously. 


\subsection{Probability of collision}

Collisions will occur when several sensor nodes try to access a common medium at the same time. LA-MAC protocol reduces the number of sensor nodes in each AS, and hence, the number of collisions will be reduced in comparison with the S-MAC protocol. Concerning the proposed CLA-MAC method, since the further number of competitive sensor nodes is reduced within each AS and even sometimes there is no need to competition, the collision rate will decrease significantly. Fig.9 and Fig.10 illustrate and compare the collision rate between two algorithms under two different situations. In the Fig.10, we have considered that the number of ASs is fixed and equal to 3. As it can be seen in Fig. 10 that in the proposed algorithm, the Probability of collisions is fixed and near to zero. As shown in Fig.9, after about AS equals to 5, the collision decrease will stop since the request for packets is distributed among ASs; as a result, the probability of collision for this type of traffic is reduced and near to zero. In both protocols, probability of collision decreases with an increase in the number of ASs and a decrease in traffic load as given in Fig.9 and Fig.10 respectively. Further, the probability of collision can be reduced by forming more number of clusters in CLA-MAC protocol.

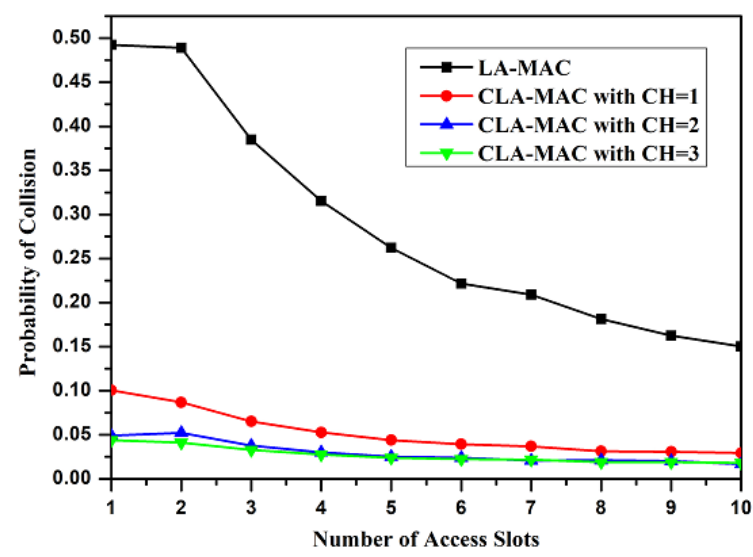

Fig. 9. Probability of collision with $T_{I A T}=5$ second

In the two figures, the differences are remarkable. Because, in the LA-MAC protocol, the competition between sensor nodes is significantly higher in comparison to CLA-MAC protocol. As a result, in the proposed CLA-MAC algorithm, the probability of packet collision is significantly reduced, and the number of successfully transmitted packets is increased. In the proposed method, the concept of the CSMA/CA technique is utilized to perform communication within each sub-group of every cluster. A schedule based slot assignment technique is used to provide an AS for each sensor nodes within a sub-group between 1 to $\mathrm{M}$. By doing this, the traffic load into their corresponding AS is reduced by a factor $\mathrm{M}$ and hence the energy-efficiency and throughput performance of proposed CLA-MAC protocol is improved. A trade-off between throughput, delay, and energy consumption concerning the variable number of ASs is revealed from the analysis as presented earlier in this section. In the proposed CLAMAC protocol, higher energy efficiency is achieved by reducing both idle-listening time and probability of collision with an increasing number of ASs due to a smaller number of retransmissions. At the same time, it can be seen that the average packet delay increases as the number of ASs increases due to the buffering of packets until the assigned slot starts. 


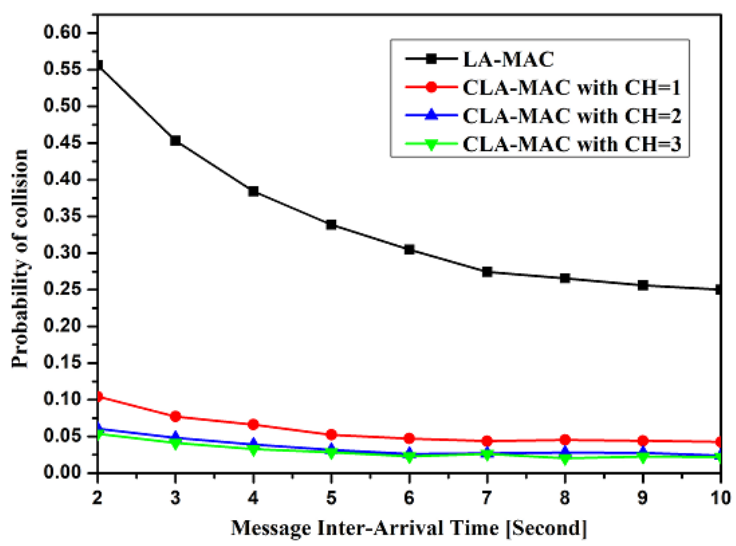

Fig.10. Probability of collision with AS $=3$

Also, the network throughput increases with an increasing number of ASs. This trade-off shows how the performance of delay is a sacrifice to reduce energy consumption and to enhance the throughput performance of the network.

\section{Conclusions}

Sensor nodes in WSNs in IoT scenario have notable limitations such as power supply, processing and memory. Also, Medium access control sub-layer manages node access to common medium. Thus, it is concluded that this MAC sub-layer plays a significant role in saving energy. Indeed, researchers are trying to design energy-efficient protocols in order to increase the network lifetime. To resolve the problems of existing approaches, in this paper, we have introduced a new algorithm for MAC sub-layer. This algorithm combines the techniques of load balancing and clustering. Simulation results indicates that the proposed approach reduces delay and collision significantly. Also, the majority of occurred events in the network are sent to the destination. Whereas the power consumption of sensor nodes in the proposed CLA-MAC method is spent for transmitting packets, energy in the LA-MAC protocol is spent for resending packets and listening to channel. We can use smarter and adaptable clustering approaches to reduce energy consumption, collision and delay in future works.

\section{References}

[1] Yinbiao, Shu, et al., Internet of things: wireless sensor networks, White Paper, International Electrotechnical Commission, Available from: http://www. iec. Ch, 2014: 11.

[2] Mainetti, Khalil, Nacer, et al., Wireless sensors networks for Internet of Things, ninth international conference on Intelligent sensors, sensor networks and information processing (ISSNIP), IEEE, 2014.

[3] Adhikari, Ratnadip. A meticulous study of various medium access control protocols for wireless sensor networks, Journal of Network and Computer Applications 41, 2014: 488-504.

[4] Bougard, Bruno, et al., Energy efficiency of the IEEE 802.15. 4 standard in dense wireless microsensor networks: Modeling and improvement perspectives, Proceedings of the conference on Design, Automation and Test in Europe-Volume 1. IEEE Computer Society, 2005. 
[5] Jha, Manish Kumar, et al., An energy-efficient multi-layer MAC (ML-MAC) protocol for wireless sensor networks, AEU-International Journal of Electronics and Communications 65.3, 2011: 209-216.

[6] Jang B. et al, An Asynchronous Scheduled MAC Protocol for Wireless Sensor Networks, Computer Networks, 2013, No. 57, Vol. 1, pp 85-98.

[7] Dinh T. et al, L-MAC: A Wake-up Time Self-Learning MAC Protocol for Wireless Sensor Networks, Computer Networks, 2016, vol. 105, pp 33-46.

[8] Bhatia A. et al, TRM-MAC: A TDMA-based Reliable Multicast MAC Protocol for WSNs with Flexibility to Trade-off between Latency and Reliability, Computer Networks, 2016, vol. 104, pp 79-913.

[9] Raja, P., and P. Dananjayan, Game theory based energy efficient hybrid MAC Protocol for lifetime enhancement of wireless sensor network, Iranian Journal of Electrical and Electronic Engineering 10.1, 2014: 10-17.

[10] Crosby, Garth V., and Farzam Vafa., Wireless sensor networks and LTE-A network convergence, Local Computer Networks (LCN), 2013 IEEE 38th Conference On. IEEE, 2013.

[11] Laya, Andres, Luis Alonso, and Jesus Alonso-Zarate, Is the Random Access Channel of LTE and LTE-A Suitable for M2M Communications? A Survey of Alternatives, IEEE Communications Surveys and Tutorials 16.1, 2014: 4-16.

[12] Liu, Yi, et al., Design of a scalable hybrid MAC protocol for heterogeneous M2M networks, IEEE Internet of Things Journal 1.1, 2014: 99-111.

[13] Aijaz, Adnan, et al., Energy-efficient uplink resource allocation in LTE networks with M2M/H2H coexistence under statistical QoS guarantees, IEEE Transactions on Communications 62.7, 2014: 2353-2365.

[14] Banerjee, Bitan, et al., BSMAC: A hybrid MAC protocol for IoT systems, Global Communications Conference (GLOBECOM), IEEE, 2016.

[15] Roy, Swati Sucharita, et al., Building a Sustainable Internet of Things: Energy-Efficient Routing Using Low-Power Sensors Will Meet the Need, IEEE Consumer Electronics Magazine 7.2, 2018: 42-49.

[16] Hamrioui, Sofiane, and Pascal Lorenz., Efficient medium access protocol for Internet of things applications, International Journal of Communication Systems 30.10, 2017: e3227.

[17] Kiran, M. Pavana Ravi Sai, Y. R. V. Prasad, and Pachamuthu Rajalakshmi., Modeling and Analysis of IEEE 802.15. 4 Multi-hop Networks for IoT Applications, Wireless Personal Communications 100.2, 2018: 429-448.

[18] Mroue, Hussein, et al., MAC layer-based evaluation of IoT technologies: LoRa, SigFox and NB-IoT, Communications Conference (MENACOMM), IEEE Middle East and North Africa. IEEE, 2018.

[19] Kumarawadu, Priyantha, et al., Algorithms for node clustering in wireless sensor networks: A survey, Information and Automation for Sustainability, 2008. ICIAFS 2008. 4th International Conference on. IEEE, 2008.

[20] Rigazzi, Giovanni, et al., Aggregation and trunking of M2M traffic via D2D connections, Communications (ICC), 2015 IEEE International Conference on. IEEE, 2015.

[21] Sadek, Rowayda A., Hybrid energy aware clustered protocol for IoT heterogeneous network, Future Computing and Informatics Journal, 2018.

[22] Heinzelman, Wendi Rabiner, Anantha Chandrakasan, and Hari Balakrishnan. "Energy-efficient communication protocol for wireless microsensor networks." In Proceedings of the 33rd annual Hawaii international conference on system sciences, pp. 10-pp. IEEE, 2000.

[23] R. Rom and M. Sidi, Multiple access protocols: performance and analysis. Springer Science and Business Media, 2012. 


\section{Authors' Profiles}

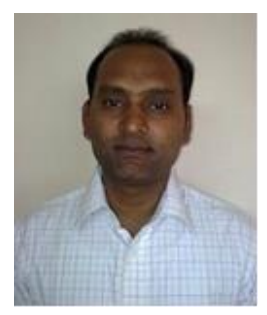

Atul Kumar Pandey Atul Kumar Pandey received the Bachelor of Technology degree in Electronics and Communication Engineering from Uttar Pradesh Technical University, Lucknow, India in 2007 and Master of Engineering degree in Electronics and Communication Engineering from Birla Institute of Technology, Mesra, Ranchi, India in 2009. He currently is an Assistant Professor in the Department of Electronics and Communication Engineering, Birla Institute of Technology, Extension Centre Patna, Patna, India. His research interests are Wireless Communication, Digital communication, Wireless sensor networks and Internet of Things (IoT).

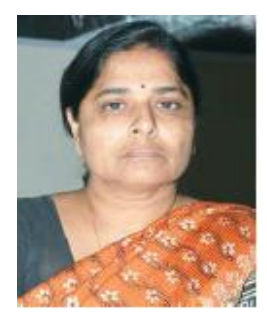

Nisha Gupta Nisha Gupta Nisha Gupta received the Bachelors and Masters degrees in Electronics and Telecommunication and Electrical and Electronics engineering both from Birla Institute of Technology, Mesra, Ranchi, India and Ph.D. degree from the Indian Institute of Technology, Kharagpur, India. She was a post-doctoral fellow at University of Manitoba, Canada from 1997-1998 before joining the department of Electronics and Communication Engineering, Birla Institute of Technology in 1999 as a Reader. Currently, she is a Professor in the same department. She has authored and co-authored more than 100 technical journal articles and conference papers. Her research interests are Computational Electromagnetics, Antennas for Wireless Communication, AI techniques in Wireless, Mobile Communication and EMI/EMC and Wireless sensor networks.

How to cite this paper: Atul Kumar Pandey, Nisha Gupta, "An Energy Efficient Clustering-based Load Adaptive MAC (CLA-MAC) Protocol for Wireless Sensor Networks in IoT", International Journal of Wireless and Microwave Technologies(IJWMT), Vol.9, No.5, pp. 38-55, 2019.DOI: 10.5815/ijwmt.2019.05.04 